\title{
MicroReview
}

\section{Transcription antitermination: the $\lambda$ paradigm updated}

\author{
David I. Friedman ${ }^{1} *$ and Donald L. Court ${ }^{2}$ \\ 'Department of Microbiology and Immunology, University \\ of Michigan Medical School, Ann Arbor, Michigan 48109- \\ 620, USA. \\ ${ }^{2}$ Laboratory of Chromosome Biology, ABL-Basic \\ Research Program, NCl-Federick Cancer Research and \\ Development Center, PO Box B, Frederick, Maryland \\ 21702, USA.
}

\section{Summary}

Coliphage $\lambda$ employs systems of transcription termination and antitermination to regulate gene expression. Early gene expression is regulated by the phage-encoded $\mathbf{N}$ protein working with a series of Escherichia coli proteins, Nus, at RNA sites, NUT, to modify RNA polymerase to a termination-resistant form. Expression of $\lambda$ late genes is regulated by the phage-encoded $Q$ antitermination protein. $Q$, which appears to use only one host factor, acts at a DNA site, qut, to modify RNA polymerase to a terminationresistant form. This review focuses on recent studies which show that: (i) $\mathrm{N}$ can mediate antitermination in vitro, independent of Nus proteins. (ii) Early genes in another lambdoid phage HKO22 are also regulated by antitermination, where only an RNA signal appears necessary and sufficient to create a terminationresistant RNA polymerase. (iii) A part of the qut signal appears to be read from the non-template DNA strand. (iv) A host-encoded inhibitor of $\mathrm{N}$ antitermination appears to act through the NUT site as well as with the $\alpha$ subunit of RNA polymerase, and is antagonized by NusB protein.

\section{Introduction}

The strategy of controlling gene expression by modulating transcription termination was discovered in coliphage $\lambda$ (Roberts, 1969). Roberts proposed that the $\mathrm{N}$ gene product of $\lambda$, which was known to be required for the synthesis of nearly all $\lambda$ transcripts, acts to permit transcripts initiating at the early $\lambda$ promoters to override Rho-dependent

Received 27 March, 1995; revised 15 June, 1995; accepted 20 June, 1995. This review is dedicated to the memory of Dr Harrison Echols who served as an inspiration to all in the lambda field. *For correspondence. Email davidfri@umich.edu; Tel. (313) 7633142 Fax (313) 7643562 termination signals. This proposal was based on his studies identifying Rho-dependent termination signals in $\lambda$ as well as on a wealth of well-characterized mutants and variants of $\lambda$. Following this seminal proposal, in vivo and in vitro studies contributed to a more sophisticated understanding of $\mathrm{N}$-mediated transcription antitermination. Moreover, additional regulatory systems based on transcription termination were identified in $\lambda$ (Roberts, 1992), and related phages (Franklin, 1985) as well as in bacteria (Landick and Turnbough, 1992) and eukaryotes (Spencer and Groudine, 1990; Jones and Peterlin, 1994). A second class of transcription terminators, Rho- or factor-independent, has also been identified (Yager and Hippel, 1987). N-modified transcription also overrides this type of terminator (Gottesman et al., 1980; DeVito and Das, 1994).

Studies on the $\lambda$ transcription-antitermination systems continue to serve as primary sources for new insights into the process of transcription elongation, in eukaryotes as well as prokaryotes (Das, 1993; Greenblatt et al., 1993). Therefore, this review will focus on termination and antitermination regulatory systems in the lambdoid phages. Over the last few years there have been a number of excellent reviews on the topic of transcription antitermination, focusing on the well-characterized $N$ and $Q$ systems of $\lambda$ (reviews are listed before each section in the Prologue). We refer the reader to those reviews for detailed discussions of $N$ and $Q$ as well as references to the older literature. We will limit our discussion to an abbreviated summary of the material that has been previously reviewed and focus on the more recent findings hoping to update the readers on material with which they may not be familiar.

\section{PROLOGUE}

\section{The $\mathbf{N}$ system}

(Reviewed in: Das, 1992; Das, 1993; Roberts, 1993; Greenblatt et al., 1993; Greenblatt, 1992; Friedman, 1988; Friedman and Gottesman, 1983.)

Early-gene transcription of the $\lambda$ genome originates at two promoters, $\mathrm{pL}$ (leftward) and $\mathrm{pR}$ (rightward), proceeding in opposite directions (Fig. 1). Transcription on the left passes through the $\mathrm{N}$ gene and, on the right the cro gene, before encountering Rho-dependent terminators tL1 and tR1 respectively. Approximately $50 \%$ of the transcripts initiating at $\mathrm{pR}$ terminate at $\mathrm{tR} 1$, with the remainder 


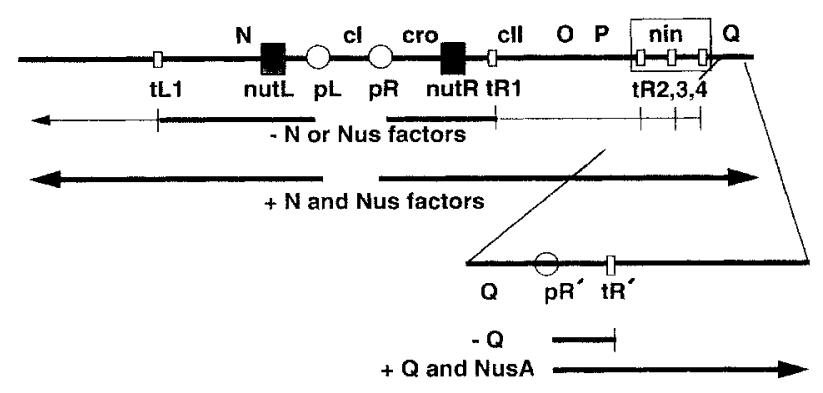

Fig. 1. Map of the regulatory region of $\lambda$ detailing transcription patterns. To orient the reader, a representative collection of genes are shown at the top of the figure. The $N$ and $Q$ genes are discussed in the text. The nin region (Court and Sato, 1969) is defined by a $2.8 \mathrm{~kb}$ deletion (Fiandt et al., 1971) that removes three regions of transcription termination (Cheng et al., 1995). The following lists the activities of the products of genes not discussed in detail in the text: repressors, $c l$ and cro; transcription activator, $c$ ll; replication, $O$ and $P$. The position of regulatory signals are shown below the listing of genes: small open boxes, terminators; large filled boxes, nut sites; open circles, promoters. Transcription patterns in the presence and absence of the $\mathrm{N}$ and $\mathrm{Q}$ antitermination proteins as well as associated Nus factors are shown beneath the map of the regulatory region.

continuing through $\mathrm{O}$ and $\mathrm{P}$ and terminating in the collection of Rho-dependent and factor-independent terminators in the $\operatorname{nin}$ region. In the presence of $\mathrm{N}$, transcription transcends these termination barriers and, consequently, more distal genes are expressed. In fact, if $\lambda$ is integrated into the bacterial chromosome, modified transcription can extend for over tens of kilobases into the bacterial chromosome, overriding a wide range of both Rho-dependent and Rhoindependent terminators.

In addition to $\mathrm{N}$, a number of host proteins, Nus, are required for $\mathrm{N}$-mediated antitermination. A role for these proteins was, in most cases, first deduced from genetic experiments with mutants that were unable to support $N$ action. Identification of the affected genes and demonstration that they were recessive showed that the mutations affect a required activity and lead to the identification of the products. In vitro studies confirmed that these products were required for $\mathrm{N}$ action. Some of the Nus factors had previously been unidentified while others are proteins of known function. NusA, a $56 \mathrm{kDa}$ essential protein, was subsequently shown to affect transcriptional pausing, termination, and antitermination. NusB, a 14 kDa protein essential for cell growth only at low temperatures, may be involved in translation as well as transcription. The nusE71 mutation, which defined the NusE product, is an allele of rpsJ, encoding ribosomal protein S10. NusD was identified as the termination factor Rho. NusG, first identified through a nusG mutation that suppressed the effects of the nusA1 and nusE 71 mutations, is a required factor for the $\mathrm{N}$ antitermination in vitro as well as an enhancer of termination factor Rho. The $\mathrm{N}$ and Nus proteins function as a complex modifying RNA polymerase to a termination-resistant form.
The N-Nus complex is assembled at sites, NUT, located downstream of the $\mathrm{pL}$ and $\mathrm{pR}$ promoters (see Figs 1 and 2). Transcription initiates at $p R$ and traverses the cro gene before reaching the nut sequence. The NUT signals, read from the RNA, have been divided into two components, BOXes $A$ and $B$, with an intervening spacer region (Fig. 2). The boxA sequence is conserved not only amongst lambdoid phages but also within the antiterminator elements of the $r r n$ operons. The BOX-B sequence forms a stem-loop structure in the RNA. In vitro studies suggest that as soon as NUT RNA is synthesized, $\mathrm{N}$ and Nus factors assemble into a complex and associate with RNA polymerase (Fig. 2). These studies also suggest that the assembled complex remains associated with the RNA polymerase as it continues transcription. The NUT RNA may be part of the complex. In this way, the polymerase is modified and retains antitermination activity as it transcribes kilobases of DNA. Both in vivo and in vitro evidence indicate that host factor NusB, in complex with NusE, binds to BOX-A, and $N$ protein binds to the loop of BOX-B.

All lambdoid phages have mechanisms for converting transcription complexes initiating at early promoters to termination-resistant forms. With one exception (to be discussed below), those studied have systems analogous to the $N$ system of $\lambda$. The two other characterized $N$ systems are those of phages 21 and P22. In those cases, $N$ genes and nut sites are located at positions on these phage genomes that are analogous to the positions of their $\lambda$ homologues. Despite these similarities, products of the $N$ genes of different lambdoid phages are specific for their cognate NUT sites under physiological conditions. The arginine-rich amino terminus of $N$ recognizes the $B O X-B$ region of NUT. A consensus boxA sequence was deduced from a comparison of boxA sequences and shown to be more effective than that found in the $\lambda$ nut region. Both in vivo and in vitro studies suggest that NusB and possibly S10 (NusE) interact with BOX-A, with the consensus BOX-A being the most effective binder.

\section{The $Q$ system}

(Reviewed in: Roberts, 1992.)

$\mathrm{N}$-modified transcription from $\mathrm{pR}$ passes through $\mathrm{tR} 1$ and the Rho-independent (tR2) and Rho-dependent (tR3 and tR4) barriers in the nin region before extending into the $Q$ gene (Fig. 1). The $Q$ gene product is the primary factor in a second $\lambda$ antitermination system allowing transcription initiating at the $\mathrm{pR}^{\prime}$ promoter, immediately beyond $\mathrm{Q}$, to extend downstream to include the lytic genes. $\mathrm{Q}$-mediated antitermination, like that of $\mathrm{N}$, requires a specific recognition signal, termed qut. However, $Q$, unlike $N$, does not bind to the RNA but binds to DNA in the region between the -10 and -35 promoter elements and interacts with 

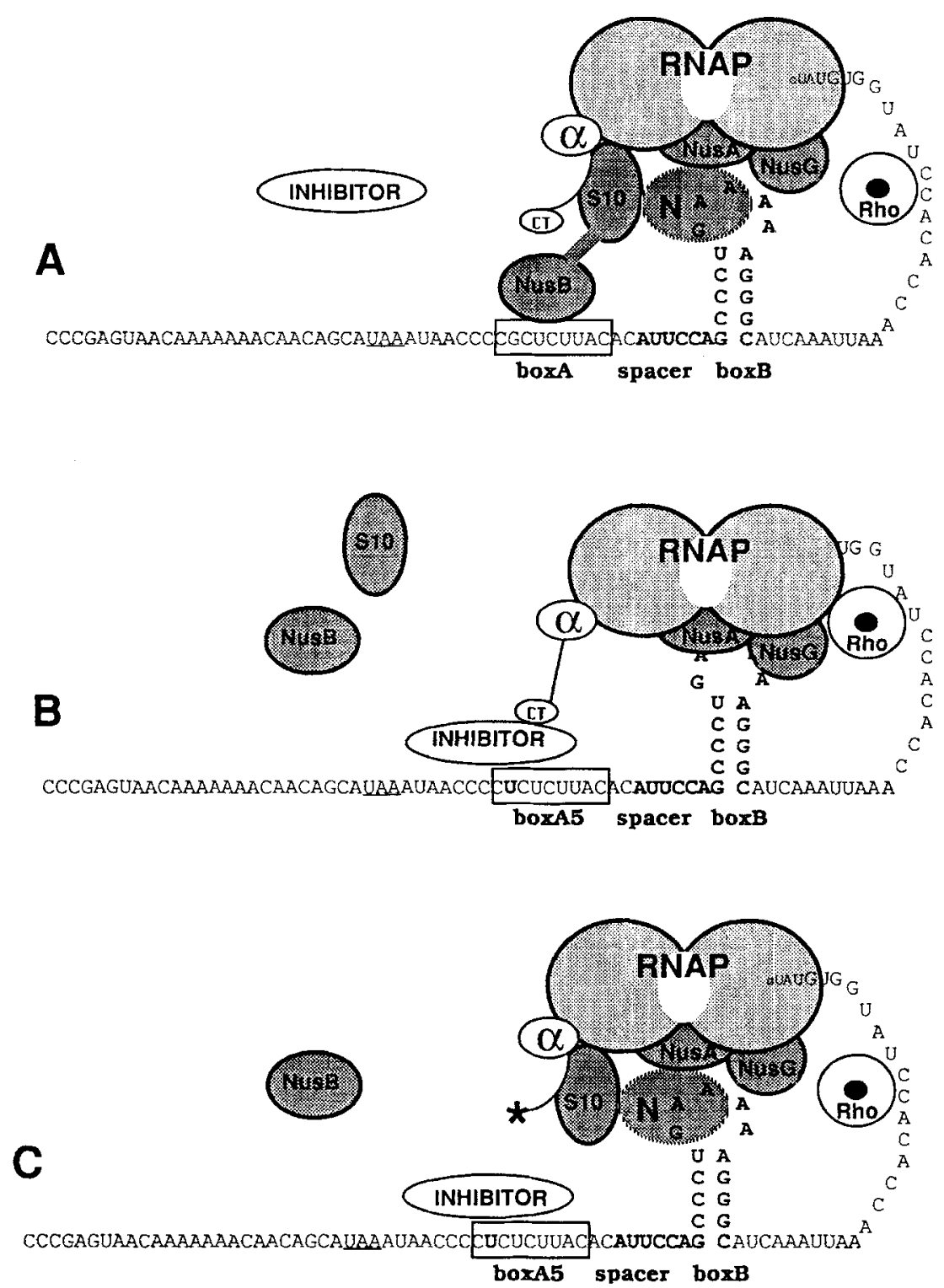

Fig. 2. Hypothetical representation of possible interactions in the formation of the $\mathrm{N}$-mediated antitermination complex under conditions where NusB either succeeds or fails to block action of the putative inhibitor. In each panel, RNA polymerase is shown associated with Nus factors at the NUT-L site. The carboxy terminus (CT) of the $\alpha$ subunit of RNA polymerase is indicated. The positions of boxes $A$ and $B$ as well as the intervening spacer are also indicated. The boxA5 mutation (shown in bold) is thought to enhance inhibitor binding.

A. The active complex may form when NusB binds to BOX-A and effectively blocks action of the inhibitor.

B. How the inhibitor may be positioned to effectively block productive complex formation by binding to BOX-A and the carboxy terminus of $\alpha$.

C. How absence of the carboxy terminus (CT) of $\alpha$ might reduce inhibitor binding to the complex.
RNA polymerase paused just downstream at +16 relative to the start of transcription. Also, unlike the $\mathrm{N}$ system, only one host factor, NusA, has been implicated in $Q$ action.

Studies with $\mathrm{Q}$, as well as $\mathrm{N}$, suggest that antiterminator activity reduces pausing by RNA polymerase and thus conceivably propels polymerase through termination sites. Reduction in pausing could be the most obvious manifestation of the ability of antiterminators to increase the kinetics of utilization of ribonucleotide substrates.

\section{The Nun termination protein}

(Reviewed in: Friedman, 1988; Das, 1993; Gottesman and Weisberg, 1995.)
Perhaps the most striking finding since the characterization of $\mathrm{N}$ antitermination is the elucidation of the action of the Nun protein encoded by lambdoid phage HKO22. Located at the analogous position on the HKO22 genome as the $\mathrm{N}$ genes of other lambdoid phages, the nun gene encodes a protein, Nun, that resembles the $N$ family of proteins. However, Nun is dispensable for $\mathrm{HKO} 22$ growth and there is no evidence that it plays any role in HK022 development, either lytic or lysogenic. Surprisingly, Nun protein acts at the NUT sites of $\lambda$, but unlike $N$ causes transcription termination not antitermination. Therefore, an HK022 lysogen excludes growth of an infecting $\lambda$ by causing premature termination of $\lambda$ transcripts. Like $N$, action of Nun requires the host Nus proteins. What is particularly striking is the specificity of the exclusion; of the lambdoid 
phages tested, only those with the nut region of $\lambda$ are excluded.

\section{NEW INFORMATION}

\section{Minimal requirements for $\mathrm{N}$-mediated antitermination}

Earlier studies using in vitro systems defined the several components of $\mathrm{N}$-mediated antitermination (reviewed in: Das, 1992; 1993; Greenblatt, 1992; Greenblatt et al., 1993). Studies characterizing antitermination through one terminator revealed a requirement for only $\mathrm{N}$ and NusA (Whalen et al., 1988). These authors suggest that the added factors provide processivity and stability to the antiterminating transcription complex. Consistent with this argument, persistent antitermination through multiple terminators required, in addition, NusB, S10 (NusE), and NusG (Mason et al., 1992a). More recently, De Vito and Das (1994) demonstrated efficient antitermination over short distances with $\mathrm{N}$ alone, but only if $\mathrm{N}$ was supplied at high concentrations. They also showed that persistent antitermination through a number of terminators over longer distances required, in addition to $\mathrm{N}$, NusA, S10 (NusE), and NusG. Strikingly, NusB was not required for reasonable levels of persistent antitermination, although it was required for maximally effective levels. These results suggest that the essential component directing antitermination is $\mathrm{N}$ and that NusB is the most dispensable for persistent antitermination in vitro.

Studies by Chattopadhyay et al. (1995) employing mobility-shift- and RNase-protection experiments provided evidence that $\mathrm{N}$ binds to the 5 leading side of the stem and loop portion of BOX-B. In addition, these studies demonstrated that some changes in the sequence of the $B O X-B$ loop interfere with $\mathrm{N}$ binding and antitermination, while other changes do not affect $N$ binding but do interfere with the minimal $\mathrm{N}-\mathrm{NusA}$ antitermination reaction. These observations led Chattopadhyay et al. (1995) to propose that through an association of both $N$ and NusA with BOX-B, NusA helps to bring $N$ in contact with RNA polymerase to create the antitermination complex. At higher $\mathrm{N}$ concentrations, the requirement for NusA is eliminated (DeVito and Das, 1994).

We emphasize that although the $\mathrm{N}$ system in vivo requires a number of proteins in addition to $N, N$ in conjunction with a BOX-B stem and loop structure is sufficient to convert RNA polymerase to a termination-resistant form. If antitermination can be made so simple, obvious questions raised by this work are why in vivo is the $\mathrm{N}$ system complicated by so many factors, and is a complicated system necessary? We will argue that the added factors permit multiple levels of regulation through $\mathrm{N}$-mediated antitermination and review newly identified components of the $\mathrm{N}-$ Nus system that offer the possibilities of additional levels of regulation. We will also review studies characterizing other antitermination systems of lambdoid phages that suggest that simpler systems also exist.

\section{Regulation of $\lambda \mathrm{N}$ gene expression}

The focus of studies on $N$ has primarily been directed toward understanding its role as an antitermination protein with little effort directed toward understanding the regulation of $\mathrm{N}$ expression. Classically, it had been shown that $\mathrm{N}$, the first gene transcribed from $\mathrm{pL}$, is controlled by repressor and Cro proteins, both of which bind to operator sites, and respectively shut off or turn down transcription from $\mathrm{pR}$ and $\mathrm{pL}$ (Ptashne, 1992). $\mathrm{N}$ levels are also controlled post-translationally by the Lon protease, which rapidly degrades the $N$ protein (Gottesman et al., 1981).

Recent studies have shown that $\mathrm{N}$ can be further controlled at the level of translation by the RNA endonuclease RNase III (Kameyama et al., 1991). The AUG start of $N$ is located 223 nucleotides $(\mathrm{nt})$ from the start of transcription. This long RNA leader contains the NUT-L site, encompassing nt 34-64. Between NUT-L and the AUG start is a large base-paired stem-loop structure that abuts the $N$ ribosome-binding site and inhibits initiation of translation. RNase III cleaves the structure, opening the ribosomebinding site to allow more efficient translation of $N$. Since RNaselll has a regulatory role in $E$. coli (Court, 1993), it seems likely that $\lambda$, through $N$ expression, has added this regulatory scheme to the already large panoply of regulatory mechanisms operating on transcription from its early promoters.

\section{HK022 Nun protein}

Building on the earlier observations that HK022 Nun protein acted at $\lambda$ NUT sites to terminate transcription from early $\lambda$ promoters, Baron and Weisberg (1992) showed that both the boxA and boxB components of the $\lambda$ nut sequences are required for maximal Nun-mediated transcription termination. However, results of this mutational analysis also suggested that although there is significant overlap in the recognition of $\mathrm{N}$ and Nun, the recognition sequences in nut are not identical. Using a suppressor tRNA located immediately downstream from the $\lambda$ nut $L$ sequence as the reporter, Sloan and Weisberg (1993) showed that, in vivo, Nun reduced the level of biologically active suppressor IRNA and that most of the RNA terminated in a region about 100 nt downstream of nutL.

Also, similar to $\mathrm{N}$, Nun action requires host Nus proteins. Mutations in Escherichia coli nus genes that cause a failure in N-mediated antitermination also affect Nun-mediated termination (Robert et al., 1987; Robledo et al., 1990). Moreover, other mutations in these nus genes have been isolated that reduce Nun action but not $\mathrm{N}$ action (Robledo 
et al., 1991). Selected as inhibitors of Nun termination at NUT-R, surprisingly, the latter mutations have no effect on Nun termination at NUT-L. Identification of a host mutation, rpoC100, that interferes with Nun-directed termination suggests a role for the $\beta^{\prime}$ subunit of RNA polymerase in transcription termination (Robledo et al., 1991).

In vitro experiments show that, as does N, Nun functions efficiently in the presence of all of the purified Nus factors, but in this case transcription arrest is the end result (Hung and Gottesman, 1995). Moreover, Nun also functions in a minimal system with BOX-B, independently of Nus factors.

Chimeric proteins expressed from hybrid genes created by in vitro splicing of $\mathrm{N}$ and nun genes have been used to relate function and structure of Nun (K. Henthorn and D. Friedman, in preparation). The proteins expressed from these genes were tested for termination and/or antitermination activity. These experiments, that show that the RNA-binding domains of $\mathrm{N}$ and Nun are exchangeable, are consistent with the RNA-recognition element being located in the amino-terminal portion and a transcriptionregulating element being located in the carboxy portion of Nun. This assignment is consistent with previous studies showing that the RNA-binding domain of $\mathrm{N}$ is in the aminoterminal region of that protein (Lazinski et al., 1989; Franklin, 1993). One chimera, having the amino $3 / 4 \mathrm{~N}$ protein and the carboxy $1 / 4$ Nun protein, exhibits the unusual property of blocking antitermination when $r p o C$ is wild type, and promoting antitermination with the rpoC100 mutant allele that normally interferes with Nun termination. This observation strengthens the argument that interactions with the $\beta^{\prime}$ subunit may play an important role in determining the elongation mode of RNA polymerase.

\section{Antitermination in HKO22}

HK022 early-gene organization is similar to that of $\lambda$, and also like $\lambda$, HK022 requires transcription antitermination for expression of its $Q$ gene. However, we remind the reader that the nun gene, while occupying a position on the HK022 genome analogous to the position of $N$ on the $\lambda$ genome, does not appear to function as an antitermination protein (Robert et al., 1987). Although there is no evidence that $\mathrm{HKO22}$ has an antitermination function analogous to $\lambda \mathrm{N}$, there are sites downstream of the early promoters that have similar sequences and are required for transcription antitermination. These common sites, composed of two sequences called box 1 and box2, have dyad symmetry and the potential to form a stem and loop structure in the RNA (Oberto et al., 1993). These sequences, however, appear otherwise unrelated to the box $A$ and box $B$ sequences of the nut regions of $\lambda$ and other lambdoid phages. More surprisingly, an HK022-encoded protein does not appear to be necessary when a termination-resistant transcription complex is formed by RNA polymerase transcribing the box1 and box2 region of HKO22.

If the only contribution of $\mathrm{HKO22}$ to this transcription antitermination reaction is the RNA sequence, is there then an $\mathrm{N}$-like factor expressed by the host that acts at or near BOX 1 and BOX 2? Weisberg and co-workers (Clerget et al., 1995) addressed this question by looking for $E$. coli mutants that fail to support this antitermination. The only mutations identified were in rpoC. No evidence for Nus-like or $\mathrm{N}$-like factors was uncovered. One explanation for these results is that a protein is not required for antitermination from the early promoters of HK022, but rather it is the interaction of RNA sequences with RNA polymerase that produces the termination-resistant complex. If the RNA always assumes the same structure after transcription, then it is possible that transcription from the HK022 early promoters always antiterminates, without the delay required for $\mathrm{N}$ systems. This raises an interesting question related to $\mathrm{N}$-mediated antitermination. Is the NUT RNA the ultimate determinant for antitermination, with the various proteins, including $\mathrm{N}$, only playing supporting regulatory roles?

\section{Q function}

Most of our knowledge of $Q$ action derives from in vitro studies from the Roberts laboratory. They have focused their efforts on the $Q$ genes of $\lambda$ and its relative, phage 82. Their studies indicate that RNA polymerase binds at $\mathrm{pR}^{\prime}$ and proceeds to pause sites located, relative to the transcription start, at +16 or 17 in $\lambda$ (Grayhack et al., 1985) and +25 in 82 (Goliger and Roberts, 1989). Polymerases, paused at these sites, are the substrate for $Q$. Mutational analysis and DNA footprinting studies indicate that the $\lambda Q$ recognition site, qut, may span a segment from within the $\mathrm{pR}^{\prime}$ promoter to +9 relative to the transcription start (Yarnell and Roberts, 1992). For this reason qut and $Q$ action cannot be separated from the $p R^{\prime}$ promoter. This is unlike $\mathrm{N}$, where unrelated promoters function effectively in $\mathrm{N}$-mediated antitermination providing that the nut site is promoter distal and placed upstream of any terminators (De Crombrugghe et al., 1979).

$\lambda \mathrm{pR}^{\prime}$ DNA templates, mutant at positions +2 and +6 , reduce RNA polymerase pausing and prevent $Q$ binding to the antitermination complex. Heteroduplex DNA containing these same changes but only in the non-template (non-transcribed) strand exert the same effect, whereas heteroduplexes with these changes only in the template (transcribed) strand have little, if any, effect on pausing or $Q$ function (Ring and Roberts, 1994). We emphasize the point that this signal is recognized in the nontranscribed DNA strand and not the RNA or the transcribed DNA strand. Since RNA polymerase incorporating the 16 th nt would cause the DNA region including +2 and 
+6 to be melted and thus become part of the transcription bubble, the non-transcribed strand could be free to interact with RNA polymerase to cause pausing, a mechanism which is suggested by heteroduplex analysis as well as in vivo- and in vitro-footprint studies. Pausing of polymerase at +16 is not sufficient to foster $Q$ interaction, since a polymerase can be paused artificially at +16 on DNA templates, mutant at +2 or +6 , and there is no $Q$ binding (Yarnell and Roberts, 1992). Thus, either $Q$ itself recognizes these positions in the non-transcribed strand or $Q$ recognizes a form of RNA polymerase that is altered by an interaction with nucleotides in the nontranscribed strand at these positions to facilitate pausing and interaction with $\mathrm{Q}$.

$Q$ is distinquishable from $N$ in another important way; it is an example of an antitermination protein that acts without an array of other proteins (Barik and Das, 1990), requiring only one additional protein, NusA (Grayhack et al., 1985). Moreover, that requirement does not appear to be absolute (Goliger and Roberts, 1989). Thus, Q protein, with a qut site, is sufficient to cause antitermination, and NusA only stimulates the effect. Studies with an E. coli carrying a nusA gene inactivated by a deletion-insertion, discussed in detail below, confirms that Q-mediated antitermination is effective without NusA (C. Zheng, D. L. Court, and D. I. Friedman, unpublished). Importantly, $\lambda$ grows in this host, while a $\lambda Q^{-}$derivative fails to grow, indicating a requirement for $Q$ action even in the absence of any NusA. In some ways, the $Q$ system resembles the minimal $N$ system, where high concentrations of $N$ are sufficient for antitermination in the absence of Nus factors. The difference, however, is that the $Q$ system is more processive than the minimal $\mathrm{N}$ system. Perhaps the basis for this difference resides in two elements that may be specific for $Q$, the paused RNA polymerase intermediate at the qut site, and the involvement of the nontemplate DNA strand.

\section{The inhibitor}

One focus of this review has been the complexity of the $N$ antitermination system which is influenced by a large number of host proteins. This stands in distinct contrast to the simpler $Q$ system that is modulated by, perhaps, one additional protein, and the even simpler early antitermination system of HKO22 that apparently requires only an RNA sequence. This complexity takes an added turn when we now examine the possibility of an additional factor that inhibits $\mathrm{N}$ action. The hypothesis that a factor exists that acts at BOX-A to inhibit $\mathrm{N}$-mediated antitermination (Patterson et al., 1994) emerged from two lines of apparently contradictory evidence. First, it was found that point mutations in boxA (boxA5 or boxA16) inactivate the nut site (Olson et al., 1984; Robledo et al., 1990). Second, it was found that deletions that included box $A$ appear to have little effect on antitermination through Rho-dependent and Rho-independent termination signals (Patterson et al,, 1994; Peltz et al., 1985; Zuber et al., 1987). Moreover, nut sites with those deletions direct antitermination independently of NusB, while still requiring $\mathrm{N}$ and the other Nus factors. These paradoxical observations can be explained by postulating that an inhibitory molecule recognizes BOX-A and that NusB competitively blocks the action of the inhibitor in wild-type situations (Fig. 2). Moreover, as a corollary, it was postulated that a nut region with the BOXA-5 mutation is a preferred target for the inhibitor, explaining why a deletion of boxA is less detrimental to $\mathrm{N}$-mediated antitermination than is the boxA5 mutation. Although this proposed role for NusB action was based more on deduction than direct evidence, subsequent studies have lent significant credence to the model.

We discuss four results supporting the model and its corollary. First, high level expression of transcripts from a plasmid containing a nut region with the box $A 5$ mutation suppressed the nusB5 mutation for growth of $\lambda$ (Patterson et al., 1994). It was argued that excess boxA5 transcripts selectively titrate a diffusible inhibitor, lowering its functional levels, and thereby freeing other NUT sites on $\lambda$ transcripts from inhibitor and reducing the requirement for NusB. Second, the nusB101 mutation suppresses the $N$ antitermination defects of mutations in the nus $A$ and $E$ genes in a boxA-dependent manner (Court et al., 1995). As described above, in a nus ${ }^{+}$host, the boxA region of nutR can be deleted without preventing antitermination. but the suppressor activity of nusB101 is not observed if the boxA sequence is deleted. Third, NusB and S10 are required for the in vitro-antitermination reaction controlled by the BOX-A sequence of the leader region of the $r r n$ operons of $E$. coli (Squires et al, 1993) and bind specifically to the consensus BOX-A RNA sequence from an rrn leader region (Nodwell and Greenblatt, 1993). Fourth, DeVito and Das (1994) argue that the dispensability of NusB for persistent in vitro antitermination in a purified system is consistent with its role as an antagonist of an inhibitory function; in the in vitro reaction where there appears to be no inhibitor, NusB is not needed for effective antitermination.

\section{Role of the $\alpha$ subunit of RNA polymerase}

Several transcription factors in E. coli (e.g., Crp, Fnr, IHF, and OmpR-EnvZ, as well as the phage P2 Ogr protein) exert their action through contact with the $\alpha$ subunit of RNA polymerase (reviewed in: Ishihama, 1992; Russo and Silhavy, 1992; Busby and Ebright, 1994). These interactions have been identified by mutations in rpoA, the gene encoding the $\alpha$ subunit. Different point mutations located in different positions of the carboxy-terminal domain (CTD) of rpoA show specificity by blocking the

(19) 1995 Blackwell Science Ltd, Molecular Microbiology, 18, 191-200 
action of specific transcription factors. This suggests that different activators contact different amino acid patches of the carboxy domain of $\alpha$. A variant $\alpha$ deleted for the entire carboxy terminus has a general defect for all of the transcription factors tested.

A newly identified $r p O A$ mutation, $D 305 E$, suppresses the effects of nus mutations (nUSA1, nusB5, and nusE71) on $\mathrm{N}$ action (A. Schauer, S.-W. Cheng, L. St. Pierre, D. Alessi, D. Hidayetoglu, D. Court, and D. I. Friedman, submitted). The rpoAD305E mutation also changes an amino acid in the carboxy terminus of $\alpha$. Initially, it was assumed that rpoAD305E might enhance an interaction of one of the components of the N-Nus complex with RNA polymerase, in this way compensating for an ineffective interaction of the mutant Nus factors. Surprisingly, a truncated $\alpha$, missing the CTD, exhibits suppression very similar to that by the D305E point mutation. If the mechanism of suppression by the point and deletion mutations are similar, then it is difficult to conceive how this would occur by an enhanced interaction favouring antitermination. Rather, under Nus ${ }^{-}$conditions, the carboxy terminus of $\alpha$ appears inhibitory for $\mathrm{N}$-mediated antitermination.

How then do the rpoA mutations suppress the effect of nus mutations? Taking into consideration what we know about the $\alpha$ subunit and the $\mathrm{N}$ system, our attention is directed toward two types of sites on the $\lambda$ genome. The first are the early $\lambda$ promoters that are located upstream of the nut sites. An argument favouring these promoters as the responsible sites can be made on the basis of the in vivo studies showing that the wide array of contacts $\alpha$ makes with proteins or DNA that play important roles in stimulating transcription, are at promoters. In fact, Giladi et al. (1992) have shown in vitro that $\alpha$ plays a regulatory role at the $\mathrm{pL}$ promoter. Perhaps an interaction at the early $\lambda$ promoters mediated through $\alpha$, altering RNA polymerase per se or the rate of initiation, ultimately reduces the effectiveness of $\mathrm{N}-\mathrm{Nus}$ complex formation at the nut site. Accordingly, the $\alpha$ mutations might interfere with the interaction at the promoter and thus facilitate complexformation downstream at the nut site. This facilitated formation of $\mathrm{N}$ antitermination complexes would allow suppression of mutations that reduce $\mathrm{N}$ antitermination.

The second are NUT RNAs, the sites of N-Nus-RNA polymerase complex assembly. In the previous section, we discussed a putative inhibitor of $\mathrm{N}$-mediated antitermination. Perhaps this inhibitor, positioned by BOX-A RNA, also interacts with rpoA (Fig. 2B). Deletion of the carboxy terminus of $\alpha$ or deletion of the boxA region prevent interaction of the inhibitor with $\alpha$ (Fig 2C). This model provides a unifying explanation of how $\alpha$ and the inhibitor might be involved in $\mathrm{N}$-mediated antitermination. An obvious candidate for the inhibitor is the $\alpha$ CTD itself. This seems unlikely for two reasons. First, the fact that inhibitor activity can be titrated (Patterson et al., 1994) means that the inhibitor does not act as a component of RNA polymerase. Second, overproduction of $\alpha$ has little effect on $\lambda$ growth in vivo (L. St. Pierre, D. Court and D. Friedman, unpublished).

Recent work suggests that the $\alpha$ subunit may also contact the nascent RNA strand through its carboxy terminus during elongation (Liu and Hanna, 1995). In vitro studies, in which a modified nucleotide capable of photocrosslinking was placed at specific positions in the newly transcribed RNA, show that following photoactivation, residues as far as $44 \mathrm{nt}$ from the $3^{\prime}$ end of the nascent RNA are crosslinked to the $\alpha$ subunit. Interestingly, when NusA is added to the transcription reaction, NusA becomes crossed-linked to RNA and the crosslinking of RNA to $\alpha$ is not observed.

\section{Nus factors}

\section{NusA}

A factor that plays a role in transcriptional pausing, termination, and antitermination, NusA, is essential for $E$. coli, as shown by the identification of a number of conditional lethal mutations, including amber, ts, and cs (Craven and Friedman, 1991; Nakamura et al., 1986; Nakamura and Uchida, 1983). However, E. coli can tolerate the absence of NusA providing it also has low Rho activity (Zheng and Friedman, 1994). This was shown by the construction of an $E$. coli with a $533 \mathrm{bp}$ deletion of nusA substituted with a cat cassette (AnusA533) in a strain that also carries rho mutations that substantially reduce Rho termination activity.

Results of a number of studies on Rho and NusA action provide an explanation for the viability of this double mutant. NusA protein reduces the elongation rate of RNA polymerase (Yager and Hippel, 1987), coupling transcription and translation (Landick and Turnbough, 1992; Das and Wolska, 1984; Kung et al., 1975). Thus, translation of genes with internal Rho-dependent terminators prevents premature transcription termination by Rho (Ruteshouser and Richardson, 1989). To terminate transcription, Rho must access, unencumbered, RNA some 50-100 nt upstream of the site of termination and translocate to the paused polymerase. Bound ribosomes may either block the binding of Rho or its access to the downstream paused polymerase. Nonsense mutations uncouple translation from transcription, allowing unimpeded access of Rho to the paused polymerase leading to transcription termination (Adhya et al., 1976; Platt and Richardson, 1992; Platt, 1994). It has been proposed (Zheng and Friedman, 1994) that in the absence of NusA, as in the strain carrying $\triangle$ nusA533, RNA polymerase elongates more rapidly causing a general uncoupling of transcription and translation (Das and Wolska, 1984). This allows access by Rho to the paused polymerase, leading to premature transcription termination throughout the genome. This premature termination is lethal to the cell. However, when Rho 
activity is reduced by the compensating rho mutation, premature termination is reduced and the cell can survive.

\section{NusB and NusE}

The functions NusB and S10 (NusE) appear to play roles in both transcription and translation. These two proteins interact with each other to form a complex (Mason et al., 1992b) that binds to RNA containing the consensus BOX-A sequence (Nodwell and Greenblatt, 1993). Although such an interaction has not been demonstrated for the BOX-A sequence in the $\lambda$ nut region, biological studies indicate that NusB interacts with the $\lambda$ BOX-A sequence (Friedman et al., 1990; Patterson et al., 1994; Court et al., 1995). It has been proposed that the interaction of NusB with BOX-A can facilitate $S 10$ interaction with RNA polymerase during $\mathrm{N}$ antitermination (Patterson et al., 1994) (Fig. 2A). However, it is clear that both in vivo and in vitro $S 10$ remains essential for $\mathrm{N}$-mediated antitermination reactions when boxA is deleted or NusB is not provided and thus can interact in the $\mathrm{N}$-antitermination complex independently of NusB (Patterson et al., 1994; DeVito and Das, 1994) (Fig. 2B).

$\mathrm{S} 10$ is undoubtedly a translation factor because it is a protein component of the $30 \mathrm{~S}$ ribosome subunit (Nomura and Morgan, 1977). Although NusB is not an integral ribosomal protein, it does influence translation, as shown by the observation that nus $B$ mutants have slowed translation rates relative to wild type (Taura et al., 1992), and is associated with ribosomes following high-salt wash (Das and Wolska, 1984). NusB null mutants have the interesting property of being cold sensitive for growth (Taura et al., 1992; Georgopoulos et al., 1980). At elevated temperatures, these nusB null mutants grow, but, as discussed above, exhibit reduced translation rates and a Nus ${ }^{--}$phenotype (Taura et al., 1992). The nature of the cold-sensitivity defect is not yet understood (Court et al., 1995). It is interesting to speculate that, as in the case of $\lambda$ in the absence of NusB, the proposed inhibitory function discussed in an earlier section, may prevent growth at low temperature; i.e. the inhibitor could cause the cold-sensitive phenotype.

\section{NusG and NusD (Rho)}

NusG is an essential function in $E$. coli (Sullivan and Gottesman, 1992) and is a component of the transcriptionelongation machinery ( $\mathrm{Li}$ et al., 1992). The gene was first identified by mutations that suppressed the $\mathrm{N}$-antitermination defect caused by the nusA1 mutation (Sullivan et al., 1992). In vivo studies showed that NusG is required for efficient Rho-mediated termination (Sullivan and Gottesman, 1992). Experiments with simple transcription systems confirm the in vivo observations that Rho-mediated transcription termination is enhanced by NusG and also showed that there is a proximal shift toward the promoter in the position of Rho-terminated transcripts ( $\mathrm{Li}$ et al., 1993; Nehrke et al., 1993).

During transcription elongation by RNA polymerase, the Rho hexamer binds to the free RNA, and NusG binds polymerase. Although each individually binds weakly, together they stabilize one another ( Li et al., 1993) and thereby form a more stable and effective termination complex (Nehrke and Platt, 1994). In fact, some Rho-dependent termination sites are only active in vitro in the presence of Rho and NusG (Burns and Richardson, 1995).

In vivo studies have not demonstrated a requirement for NusG in N-mediated antitermination (Sullivan and Gottesman, 1992), although purified NusG was subsequently shown to enhance elongation in a pure $\mathrm{N}$-dependent transcription-antitermination assay (Mason et al., 1992a). NusG expressed from a multicopy plasmid can rescue the NusD026 (because of a rho mutation) defect on $\mathrm{N}$ antitermination (Sullivan et al., 1992; Das et al., 1983) suggesting that the NusD mutant lacks an efficient interaction with NusG in the $\mathrm{N}$-antitermination complex. Indeed, an affinity-chromatography study showed that at higher temperatures where the wild type binds effectively, the Rho026 protein binds poorly to NusG (Li et al., 1993). We suggest that Rho is an integral component of the antitermination complex (Fig. 2) and that the Rho026 protein inhibits antitermination by compromising an important interaction of Rho and NusG within the complex.

\section{EPILOGUE}

Although the new information reviewed here has not revolutionized our thinking about $\mathrm{N}$-mediated transcription antitermination, it has contributed to a more refined understanding. It has focused attention on the $\beta^{\prime}$ subunit of polymerase as being a potentially major player in determining the fate of the transcription complex as well as the role an RNA site might play. Perhaps the most unexpected finding is the evidence suggesting a previously undescribed player, a diffusible inhibitor that antagonizes $\mathrm{N}$-mediated antitermination. The inhibitor itself has not been identified, however, the circumstantial evidence from a number of experiments provides strong support for assuming its existence. The anti-sigma factors (Duncan and Losick, 1993; Ohnishi et al., 1992) offer another example where an inhibitor is part of a regulatory scheme. Obviously, proof of this idea awaits the genetic and functional identification of the inhibitor.

What role could such an inhibitory function play? Perhaps it serves with NusB as an important cellular component of the antitermination regulatory scheme, when conditions are either less favourable for the lytic pathway or more favourable for the lysogenic pathway. By interfering with 
$\mathrm{N}$-mediated antitermination, expression of genes encoding lytic functions located downstream of the transcription terminators would be decreased.

The characterization of the antitermination mechanism in the early operons of HKO22 adds a new twist to any consideration of the role of antitermination early in phage infection. If only the HK022 RNA site is required for antitermination, then it is difficult to understand what advantage is offered to the rest of the lambdoid phages in having a special protein for antitermination. We emphasize our contention that the complex of proteins required for processive transcription antitermination by $N$ plausibly accomodate multiple pathways for regulating $\lambda$ gene expression at an early step, immediately following the first initiation event. It follows from this argument that the antitermination system in the early operons of phage HK022 is more primitive than that of other lambdoid phages, at least if possible regulatory mechanisms are considered.

We can offer several different, but not mutually exclusive, ways by which the addition of a protein component to an RNA signal for antitermination might provide further levels of regulation: (i) control of the efficiency of translation of any of the antitermination proteins, (ii) control of the rate of the degradation of the protein(s), and (iii) the competition between a protein inhibitor and antitermination factors for an RNA site. All three of these possible modes of regulation, at least under some conditions, appear to be available to influence $\lambda \mathrm{N}$-mediated antitermination.

The major question waiting to be answered is how the addition of $\mathrm{N}$ and Nus factors alters RNA polymerase so that it disregards both Rho-dependent and intrinsic termination signals.

\section{Acknowledgements}

This review is dedicated to the memory of Dr Harrison Echols who served as an inspiration to all in the lambda field. The authors apologize to all of their colleagues whose work could not be cited because of the imposed page limitation. We thank members of our laboratories for their contributions to the studies cited and to Robert Landick, Robert Weisberg, and a third, unnamed, reviewer for their thoughtful comments. Special thanks go to Asis Das for extremely helpful comments. The work from our laboratories is sponsored in part by NIH Grant Al1459-10 and The National Cancer Institute, DHHS, under contract with $\mathrm{ABL}$. The contents of this publication do not necessarily reflect the views or policies of the Department of Health and Human Services, nor does mention of trade names, commercial products, or organizations imply endorsement by the US Government.

\section{References}

Adhya, S., Gottesman, M., De Crombrugghe, B., and Court D. (1976) In RNA Polymerase. Losick, R., and Chamberlin,
M. (Eds). Cold Spring Harbor, New York: Cold Spring Harbor Laboratory Press, pp. 719-730.

Barik, S., and Das, A. (1990) Mol Gen Genet 222: 152-156. Baron, J., and Weisberg, R.A. (1992) J Bacteriol 174: 19831989.

Burns, C.M., and Richardson, J.P. (1995) Proc Natl Acad Sci USA 92: 4738-4742.

Busby, S., and Ebright, R.H. (1994) Cell 79: 743-746.

Chattopadhyay, S., Garcia-Mena, J., DeVito, J., Wolska, K., and Das, A. (1995) Proc Natl Acad Sci USA 92: 40614065.

Cheng, S.C., Court, D.L., and Friedman, D.I. (1995) Genetics, in press.

Clerget, M., Jin, D.J., and Weisberg, R.A. (1995) J Mol Biol 248: $768-780$.

Court, D. (1993) In Control of mRNA Stability. Brawerman, G., and Belasco, J. (Eds). New York: Academic Press, pp. 71-116.

Court, D., and Sato, K. (1969) Virology 39: 348-352.

Court, D.L., Patterson, T.A., Baker, N., Costantino, N., Mao, X., and Friedman, D.I. (1995) J Bacteriol 177: 2589-2591.

Craven, M.G., and Friedman, D.I. (1991) J Bacteriol 173: 1485-1491.

Das, A. (1992) J Bacteriol 174: 6711-6716.

Das, A. (1993) Annu Rev Biochem. Palo Alto, Califfornia: Annual Reviews Inc., pp. 893-930.

Das, A., and Wolska, K. (1984) Cell 38: 165-173.

Das, A., Gottesman, M.E., Wardwell, J., Trisler, P., and Gottesman, S. (1983) Proc Natl Acad Sci USA 80: 55305534.

De Crombrugghe, B., Mudryj, M., DiLauro, R., and Gottesman, M. (1979) Cell 18: 1145-1151.

DeVito, J., and Das, A. (1994) Proc Natl Acad Sci USA 91: 8660-8664.

Duncan, L., and Losick, R. (1993) Proc Natl Acad Sci USA 90: 2325-2329.

Fiandt, M., Hradecna, Z., Lozeron, H.A., and Szybalski, W. (1971) In The Bacteriophage Lambda. Hershey, A.D. (Ed.). Cold Spring Harbor, New York: Cold Spring Harbor Laboratory Press, pp. 339-354.

Franklin, N.C. (1985) J Mol Biol 181: 85-91.

Franklin, N.C. (1993) J Mol Biol 231: 343-360.

Friedman, D.I. (1988) In The Bacteriophage. Calendar R. (Ed.). New York: Plenum Publishing Corporation, pp. 263319.

Friedman, D.I., and Gottesman, M. (1983) In Lambda II. Hendrix, R.W., Roberts, J.W., Stahl, F.W., and Weisberg, R.A. (Eds). Cold Spring Harbor, New York: Cold Spring Harbor Laboratory Press, pp. 21-51.

Friedman, D.I., Olson, E.R., Johnson, L.L., Alessi, D., and Craven, M.G. (1990) Genes Dev 4: 2210-2222.

Georgopoulos, C.P., Swindle, J., Keppel, F., Ballivet, M., Bisig, R., and Eisen, H. (1980) Mol Gen Genet 179: 55-61.

Giladi, H., Igarashi, K., Ishihama, A., and Oppenheim, A.B. (1992) J Mol Biol 227: 985-990.

Goliger, J.A., and Roberts, J.W. (1989) J Mol Biol 210: 461471.

Gottesman, M.E., and Weisberg, R.A. (1995) Seminars in Virol 6: 35-42.

Gottesman, M.E., Adhya, S., and Das, A. (1980) J Mol Biol 140: $57-75$. 
Gottesman, S., Gottesman, M., Shaw, J.E., and Pearson, M.L. (1981) Cell 24: 225-233.

Grayhack, E.J., Yang, X.J., Lau, L.F., and Roberts, J.W. (1985) Cell 42: 259-269.

Greenblatt, J. (1992) In Transcriptional Regulation. McKnight, S.L., and Yamamoto, K.R. (Eds). Cold Spring Harbor, New York: Cold Spring Harbor Laboratory Press, pp. 203-226.

Greenblatt, J., Nodwell, J.R., and Mason, S.W. (1993) Nature 364: 401-406.

Hung, S.C., and Gottesman, M.E. (1995) I Mol Biol 247: $428-442$.

Ishihama, A. (1992) Mol Microbiol 6: 3283-3288.

Jones, K.A., and Peterlin, B.M. (1994) Annu Rev Biochem 63: 717-743.

Kameyama, L., Fernandez, L., Court, D.L., and Guarneros, G. (1991) Mol Microbiol 5: 2953-2963.

Kung, H., Spears, C., and Weissbach, H. (1975) J Biol Chem 250: 1556-1562.

Landick, R., and Turnbough, Jr, C.L. (1992) In Transcription Regulation. McKnight, S.L., and Yamamoto, K.R. (Eds). Cold Spring Harbor, New York: Cold Spring Harbor Laboratory Press, pp. 407-448.

Lazinski, D., Grzadzielska, E., and Das, A. (1989) Cell 59: 207-218.

Li, J., Horwitz, R., McCracken, S., and Greenblatt, J. (1992) J Biol Chem 267: 6012-6019.

Li, J., Mason, S.W., and Greenblatt, J. (1993) Genes Dev 7: 161-172.

Liu, K., and Hanna, M.M. (1995) Proc Natl Acad Sci USA 92: 5012-5016.

Mason, S.W., Li, J., and Greenblatt, J. (1992a) J Biol Chem 267: 19418-19426.

Mason, S.W., Li, J., and Greenblatt, J. (1992b) J Mol Biol 223: 55-66.

Nakamura, Y., and Uchida, H. (1983) Mol Gen Genet 190: 196-203.

Nakamura, Y., Mizusawa, S., Tsugawa, A., and Imai, M. (1986) Mol Gen Genet 204: 24-28.

Nehrke, K.W., and Platt, T. (1994) J Mol Biol 243: 830-839.

Nehrke, K.W., Zalatan, F., and Platt, T. (1993) Gene Expr 3: 119-133.

Nodwell, J.R., and Greenblatt, J. (1993) Cell 72: 261-268.

Nomura, M., and Morgan, E.A. (1977) Annu. Rev. Genet 11: 297-347.

Oberto, J., Clerget, M., Ditto, M., Cam, K., and Weisberg, R.A. (1993) J Mol Biol 229: 368-381.

Ohnishi, K., Kutsukake, K., Suzuki, H., and Lino, T. (1992) Mol Microbiol 6: 3149-3157.

Olson, E.R., Tomich, C.S., and Friedman, D.I. (1984) J Mol Biol 180: 1053-1063.

Patterson, T.A., Zhang, Z., Baker, T., Johnson, L.L., Friedman, D.I., and Court, D.L. (1994) J Mol Biol 236: 217-228.
Peltz, S.W., Brown, A.L., Hasan, N., Podhajska, A.J., and Szybalski, W. (1985) Science 228: 91-93.

Platt, T. (1994) Mol Microbiol 11: 983-990.

Platt, T., and Richardson, J.P. (1992) In Transcription Regulation. McKnight, S.L., and Yamamoto, K.R. (Eds). Cold Spring Harbor, New York: Cold Spring Harbor Laboratory Press, pp. 365-388.

Ptashne, M. (1992) A Genetic Switch. 2nd edn. Cambridge, Massachusetts: Cell Press and Blackwell Scientific Publications.

Ring, B.Z., and Roberts, J.W. (1994) Cell 78: 317-324.

Robert, J., Sloan, S.B., Weisberg, R.A., Gottesman, M.E., Robledo, R., and Harbrecht, D. (1987) Cell 51: 483492.

Roberts, J. (1992) In Transcriptional Regulation. McKnight, S.L., and Yamamoto, K.R. (Eds). Cold Spring Harbor, New York: Cold Spring Harbor Laboratory Press, pp. 389406.

Roberts, J.W. (1969) Nature 224: 1168-1174.

Roberts, J.W. (1993) Cell 72: 653-655.

Robledo, R., Gottesman, M.E., and Weisberg, R.A. (1990) J Mol Biol 212: 635-643.

Robledo, R., Atkinson, B.L., and Gottesman, M.E. (1991) J Mol Biol 220: 613-619.

Russo, F.D., and Silhavy, T.J. (1992) J Biol Chem 267: 14515-14518.

Ruteshouser, E.C., and Richardson, J.P. (1989) J Mol Biol 208: $23-43$.

Sloan, S.B., and Weisberg, R.A. (1993) Proc Natl Acad Sci USA 90: 9842-9846.

Spencer, C.A., and Groudine, M. (1990) Oncogene 5: 777785.

Squires, C.L., Greenblatt, J., Li, J., Condon, C., and Squires, C.L. (1993) Proc Natl Acad Sci USA 90: 970-974.

Sullivan, S.L., and Gottesman, M.E. (1992) Cell 68: 989-994.

Sullivan, S.L., Ward, D.F., and Gottesman, M.E. (1992) $J$ Bacteriol 174: 1339-1344.

Taura, T., Ueguchi, C., Shiba, K., and Ito, K. (1992) Mol Gen Genet 234: 429-432.

Whalen, W., Ghosh, B., and Das, A. (1988) Proc Natl Acad Sci USA 85: 2494-2498.

Yager, T.D., and Von Hippel, P.H. (1987) In Escherichia coli and Salmonella typhimurium: Cellular and Molecular Biology. Neidhardt, F.C., Ingraham, J.L., Magasanik, B., Low, K.B., Schaechter, M., and Umbarger, H.E. (Eds). Washington, D.C.: American Society for Microbiology, pp. 1241-1275.

Yarnell, W.S., and Roberts, J.W. (1992) Cell 69: 1181-1189.

Zheng, C., and Friedman, D.I. (1994) Proc Natl Acad Sci USA 91: 7543-7547.

Zuber, M., Patterson, T.A., and Court, D.L. (1987) Proc Natt Acad Sci USA 84: 4514-4518. 